\title{
Actuator Fault Diagnosis in a Boeing 747 Model via Adaptive Modified Two-Stage Kalman Filter
}

\author{
Fikret Caliskan, ${ }^{1}$ Youmin Zhang, ${ }^{2}$ N. Eva Wu, ${ }^{3}$ and Jong-Yeob Shin ${ }^{4}$ \\ ${ }^{1}$ Department of Control Engineering, Istanbul Technical University, Maslak, 34469 Istanbul, Turkey \\ ${ }^{2}$ Department of Mechanical \& Industrial Engineering, Concordia University, 1455 de Maisonneuve Boulevard W., \\ Montreal, QC, Canada H3G 1 M8 \\ ${ }^{3}$ Department of Electrical and Computer Engineering, Binghamton University (SUNY), Binghamton, NY 13902-6000, USA \\ ${ }^{4}$ Gulfstream Aerospace Corporation, Savannah, GA 31402-2206, USA
}

Correspondence should be addressed to Fikret Caliskan; caliskanf@itu.edu.tr

Received 9 November 2013; Revised 21 December 2013; Accepted 22 December 2013; Published 10 February 2014

Academic Editor: N. Ananthkrishnan

Copyright (c) 2014 Fikret Caliskan et al. This is an open access article distributed under the Creative Commons Attribution License, which permits unrestricted use, distribution, and reproduction in any medium, provided the original work is properly cited.

An adaptive modified two-stage linear Kalman filtering algorithm is utilized to identify the loss of control effectiveness and the magnitude of low degree of stuck faults in a closed-loop nonlinear B747 aircraft. Control effectiveness factors and stuck magnitudes are used to quantify faults entering control systems through actuators. Pseudorandom excitation inputs are used to help distinguish partial loss and stuck faults. The partial loss and stuck faults in the stabilizer are isolated and identified successfully.

\section{Introduction}

The Integrated Resilient Aircraft Control (IRAC) Project by NASA has conducted research to advance the state of aircraft flight control to provide on-board control resilience for ensuring safe flight in the presence of unforeseen, adverse conditions. The focus of the IRAC project research is to develop a set of validated multidisciplinary integrated aircraft control design tools and techniques for enabling safe flight in the presence of adverse conditions such as structural damage, control surface failures, icing, or aerodynamic upsets.

Aircraft have multiple control surfaces for high performance as well as redundancy. When one or more control surfaces have problems during the operation, a Fault Detection and Identification (FDI) scheme should be carried out to continue a safe flight. Figure 1 shows some basic control surfaces in aircraft. FDI methods based on analytic redundancy have been actively studied using various estimation theories. There are a number of model-based FDI methods that utilize observers, parity equations, or parameter estimation techniques.

This paper reports some results on the simultaneous estimation of the states and parameterized faults injected into the actuators of a nonlinear model of the longitudinal motion of a Boeing 747 [1], modifying the linear adaptive two-stage Kalman filter in [2]. Fault parameterization in the design model of the linear estimator aims to capture the degree at which a control surface is stuck and the extent at which the control effectiveness is lost. The estimates serve to support real-time assessment of postfailure flight envelope, in which a controlled aircraft can maintain stability and safe flight [3].

Among very rare studies, Vachtsevanos et al. [4] considered the control role portrayed by mode transitioning, envelope protection, real-time adaptation, and fault detection/control reconfiguration algorithms which are intended to safeguard unmanned aerial vehicle's integrity in the event of component failures, extreme operating conditions, or external disturbances. A fault-tolerant control system architecture was designed to combat faults in the flight control actuators. Flight test results demonstrated that the fault-tolerant architecture can accommodate stuck actuator malfunctions.

Chen and Jiang [5] investigated a linear fault-tolerant control system design technique by using an iterative learning observer when stuck actuator faults had occurred. This observer estimates both state information and stuck actuator values. The technique can accommodate multiple stuck actuators provided that enough redundant actuators exist. 


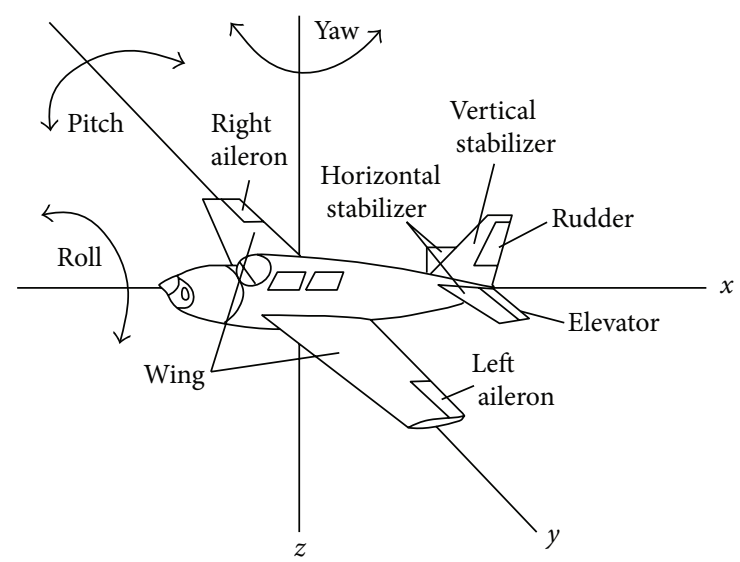

FIgURE 1: Control surfaces in aircraft.

Boskovic and Mehra [6] presented an FDI and Adaptive Reconfigurable Control (ARC) scheme for accommodation of control effector failures such as lock-in-place and hardover. The overall system consisting of online FDI observers for all control effectors guarantees asymptotic tracking and ensures convergence of the failure-related parameter estimate to its true value. The scheme can rapidly and accurately identify different control effector failures like float, hard-over, lock-in-place, and loss of effectiveness. They used adaptive interacting multiple observers to estimate the actuator faults and tested the scheme on a linearized model of the Boeing's tailless advanced fighter aircraft.

Jiang and Chowdhury [7] developed a real-time fault estimation module to estimate the actuator effectiveness. They transformed the system into two separate subsystems. One subsystem is not affected by actuator faults. The states were estimated by a reduced order Kalman filter. They demonstrated the simulation results on a linear helicopter model.

Yang and Wang [8] studied an iterative Linear Matrix Inequality- (LMI-) based stuck actuator fault detection method without capability of isolating and identifying the faults. The considered systems are modeled via multiple modes, namely, nominal case and faulty case. The actuator stuck faults are considered. A new LMI-based fault detection method for the control systems is obtained and applied to the fault detection for flight control systems.

Wang and Lum [9] developed an adaptive unknown input observer approach to detect, isolate, and identify the loss of effectiveness and stuck faults in the actuators. The approach is useful for linear systems, but the attempt to extension to nonlinear systems has not been successful [10].

The two-stage Kalman filter of Keller and Darouach [11] was applied to estimate simultaneously the state and the control effectiveness of a linear aircraft model in [2], which in addition introduced covariance-dependent forgetting factors into the filtering algorithm to accelerate convergence of the fault parameter estimates [12]. Thus, the filter acquired the name adaptive two-stage Kalman filter (TSKF). The state and control effectiveness estimates with the adaptive TSKF approach were utilized to help achieve fault-tolerant control of impaired linear aircraft model in a number of papers by the researchers such as $[13,14]$.
Amoozgar et al. [15] addressed the problem of Fault Detection and Diagnosis (FDD) of a quadrotor helicopter system in the presence of actuator faults. A TSKF is used to simultaneously estimate and isolate possible faults in each actuator. The faults are modelled as losses in control effectiveness of rotors. The developed FDD algorithm is evaluated through experimental application to an unmanned quadrotor helicopter test bed. The obtained results show the effectiveness of the proposed FDD method.

Hajiyev [16] presented an active fault-tolerant control technique against actuator/surface failures. A TSKF was designed in order to identify the control distribution matrix elements that correspond to the faulty actuator/surface; thus, the control reconfiguration was carried out using the identified control distribution matrix. The actuator/surface fault identification problem was solved through two jointly operating Kalman filters: the first one was for the estimation of the control distribution matrix elements that correspond to the faulty actuator/surface and the second one was for the estimation of the state variables of the aircraft model. In simulations, the nonlinear flight dynamics of an AFTI/F-16 fighter model was considered, and the performance of the proposed actuator/surface failure identification and reconfigurable control schemes were examined for this model.

Jiang et al. [17] presented a framework for actuator fault accommodation using a multiple model-based direct adaptive control approach that does not include explicit fault detection and isolation. The approach does not require exact information about the controlled system and persistent input excitations. Robustness is increased, and stable adaptation of unknown faults is provided. Asymptotic model following conditions and adaptive rules are derived and system stability is guaranteed, while appropriate switching of the multiple models ensures asymptotic tracking for system outputs. The efficiency of the proposed method is illustrated by simulations on an aircraft model.

This paper generalizes the design model for the linear adaptive TSKF of Wu et al. [2] which, from now on, will be referred to as a modified TSKF (MTSKF) to include stuck faults in control surfaces. The approach is applied to state and parameter estimation for a B747 nonlinear aircraft model. In order to distinguish the stuck faults from the loss of control effectiveness, persistent excitations must be present. Our findings indicate that the adaptive MTSKF can correctly estimate the aircraft states, as well as the parameters representing less severe faults near a trim point. The identification of the aerodynamic coefficients is not studied in this paper.

The paper is organized as follows. In Section 2, the linear adaptive MTSKF algorithm is described, and the linear TSKF algorithm of $\mathrm{Wu}$ et al. [2] is presented. Simulation results are given in Section 3. In conclusion, we summarize and give comments about the results.

\section{Adaptive Modified Two-Stage Kalman Filtering (MTSKF) Algorithm}

Based on the linearized model of the open-loop aircraft around a trim point, and a parameterization of two types of 
actuator faults, the following discrete time model is used as the design model of the adaptive MTSKF

$$
\begin{gathered}
x_{k+1}=A_{k} x_{k}+B_{k} u_{k}+E_{k} \gamma_{k}+B_{k} \beta_{k}+w_{k}^{x} \\
\zeta_{k+1}=\zeta_{k}+w_{k}^{\zeta}, \quad \zeta_{k}=\left[\begin{array}{ll}
\gamma_{k}^{T} & \beta_{k}^{T}
\end{array}\right]^{T} \\
y_{k+1}=C_{k+1} x_{k+1}+v_{k+1},
\end{gathered}
$$

where $x_{k} \in R^{n}, u_{k} \in R^{l}$, and $y_{k+1} \in R^{m}$ are the state, control input, and output variables, respectively. $\gamma_{k}$ and $\beta_{k}$ are bias vectors of dimension $l$, representing faults entering actuators. The noise sequences $w^{x}, w^{\zeta}$, and $v$ are assumed to be zero mean uncorrelated white Gaussian noise sequences with

$$
E\left\{\left[\begin{array}{c}
w_{k}^{x} \\
w_{k}^{\zeta} \\
v_{k}
\end{array}\right]\left[\begin{array}{lll}
w_{j}^{x} & w_{j}^{\zeta} & v_{j}
\end{array}\right]\right\}=\left[\begin{array}{ccc}
Q^{x} & 0 & 0 \\
0 & Q^{\zeta} & 0 \\
0 & 0 & R
\end{array}\right] \delta_{k j},
$$

where $Q^{x}>0, Q^{\zeta}>0, R>0$, and $\delta_{k j}$ is the Kronecker delta. The initial states $x(0)$ and $\zeta(0)$ are assumed to be uncorrelated with the white noise processes $w^{x}, w^{\zeta}$, and $v$ and have covariance $\widetilde{P}_{0}^{x}$ and, covariance $\widetilde{P}_{0}^{\zeta}$, respectively.

The components

$$
-1 \leq \gamma_{k}^{i} \leq 0, \quad j=1,2, \ldots, l
$$

of bias vector $\gamma_{k}$ describe percent reduction in control effectiveness when the terms $B_{k} u_{k}+E_{k} \gamma_{k}$ are considered together, where

$$
E_{k}=B_{k} U_{k}, \quad U_{k}=\operatorname{diag}\left(u_{k}^{1}, \ldots, u_{k}^{l}\right) .
$$

Estimator design model (1) is inherited from (3) of Wu et al. [2] with a set of new bias components

$$
\beta_{k}^{i}, \quad i=1, \ldots, l
$$

added in this paper to denote the degrees at which control surfaces are stuck. A stuck fault is modelled by the combination of the following three terms (1):

$$
B_{k} u_{k}+E_{k} \gamma_{k}+B_{k} \beta_{k}
$$

We now summarize the cases the fault parameter values represented. Specifically, no-fault case is represented by

$$
\gamma_{k}^{i}=0, \quad \beta_{k}^{i}=0 \quad i=1,2, \ldots, l
$$

$100 \gamma_{k}^{i} \%$ loss of control effectiveness in the $i$ th actuator is represented by

$$
-1 \leq \gamma_{k}^{i} \leq 0, \quad \beta_{k}^{i}=0
$$

control surface stuck at magnitude $\beta_{k}^{i}$ degrees is represented by

$$
\gamma_{k}^{i}=-1, \quad \beta_{k}^{i} \neq 0
$$

The linear adaptive TSKF of Wu et al. [2] can be directly applied to estimate both $\gamma_{k}$ and $\beta_{k}$ via generalizing $E_{k} \gamma_{k}$ to

$$
\begin{aligned}
& E_{k} \gamma_{k}+B_{k} \beta_{k}=\left[\begin{array}{ll}
E_{k} & B_{k}
\end{array}\right]\left[\begin{array}{ll}
\gamma_{k}^{T} & \beta_{k}^{T}
\end{array}\right]^{T}=\left[\begin{array}{ll}
E_{k} & B_{k}
\end{array}\right] \zeta_{k}, \\
& \text { where } \zeta_{k}=\left[\begin{array}{ll}
\gamma_{k}^{T} & \beta_{k}^{T}
\end{array}\right]^{T}
\end{aligned}
$$

which model the stuck fault as well, in addition to the loss of control effectiveness fault originally considered in $\mathrm{Wu}$ et al. [2]. Since $E_{k}=B_{k} U_{k}$,

$$
E_{k} \gamma_{k}+B_{k} \beta_{k}=B_{k} U_{k} \gamma_{k}+B_{k} \beta_{k}=B_{k}\left[U_{k} \gamma_{k}+\beta_{k}\right]
$$

therefore, the entries of $U_{k}=\operatorname{diag}\left(u_{k}^{1}, \ldots, u_{k}^{l}\right)$ must vary and evolve in time independently (persistently excited) to allow the estimator to distinguish between $\gamma_{k}$ and $\beta_{k}$ and among their components.

To make the two-stage filter algorithm more responsive to the changes of bias parameter, like the fault of parameters, following the idea in Parkum et al. [12], a bias covariance matrix related forgetting factor technique can be used in (16). Consider

$$
P_{k+1 / k}^{\zeta}=\sum_{i=1}^{l} \frac{\alpha_{k / k}^{i}}{\lambda_{k}^{i}} e_{k}^{i}\left(e_{k}^{i}\right)^{T}+Q_{k}^{\zeta}, \quad 0<\lambda_{k}^{i} \leq 1 .
$$

The forgetting factor $\lambda_{k}^{i}$ can be chosen as a decreasing function of the amount of information received in the direction $e_{k}^{i}$. Since the eigenvalue $\alpha_{k / k}^{i}$ of $P_{k / k}^{\zeta}$ is a measure of the uncertainty in the direction of $e_{k}^{i}$, a choice of forgetting factor $\lambda_{k}^{i}$ can be chosen as

$$
\lambda_{k}^{i}= \begin{cases}1, & \alpha_{k / k}^{i}>\alpha_{\max } \\ \alpha_{k / k}^{i}\left[\alpha_{\min }+\frac{\alpha_{\max }-\alpha_{\min }}{\alpha_{\max }} \alpha_{k / k}^{i}\right]^{-1}, & \alpha_{k / k}^{i} \leq \alpha_{\max } .\end{cases}
$$

The linear TSKF algorithm of Wu et al. [2] is as follows.

Bias-free state estimator is

$$
\begin{gathered}
\tilde{x}_{k+1 / k}=A_{k} \tilde{x}_{k / k}+B_{k} u_{k}+\left[W_{k}-V_{k+1 / k}\right] \widehat{\zeta}_{k / k}, \\
\widetilde{P}_{k+1 / k}^{x}=A_{k} \widetilde{P}_{k / k}^{x} A_{k}^{T}+Q_{k}^{x}+W_{k} P_{k / k}^{\zeta} W_{k}^{T}-V_{k+1 / k} P_{k+1 / k}^{\zeta} V_{k+1}^{T}, \\
\widetilde{x}_{k+1 / k+1}=\widetilde{x}_{k+1 / k}+\widetilde{K}_{k+1}^{x}\left[y_{k+1}-C_{k+1} \widetilde{x}_{k+1 / k}\right] \\
\widetilde{K}_{k+1}^{x}=\widetilde{P}_{k+1 / k}^{x} C_{k+1}^{T}\left[C_{k+1} \widetilde{P}_{k+1 / k}^{x} C_{k+1}^{T}+R_{k+1}\right]^{-1} \\
\widetilde{P}_{k+1 / k+1}^{x}=\left[I-\widetilde{K}_{k+1}^{x} C_{k+1}\right] \widetilde{P}_{k+1 / k}^{x}
\end{gathered}
$$

where the filter residual vector and its covariance are given as

$$
\begin{gathered}
\tilde{r}_{k+1}=y_{k+1}-C_{k+1} \tilde{x}_{k+1 / k}, \\
\widetilde{S}_{k+1}=C_{k+1} \widetilde{P}_{k+1 / k}^{x} C_{k+1}^{T}+R_{k+1} .
\end{gathered}
$$




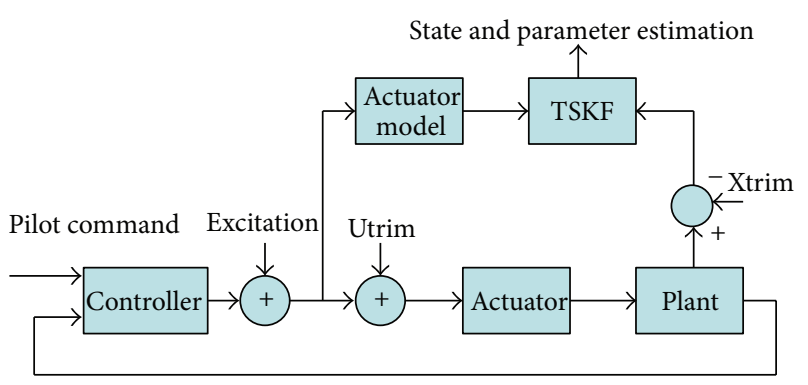

Figure 2: The overall structure of the closed-loop nonlinear B747 aircraft subject to actuator faults.

Bias estimator is

$$
\begin{gathered}
\widehat{\zeta}_{k+1 / k}=\widehat{\zeta}_{k / k}, \\
\widetilde{P}_{k+1 / k}^{\zeta}=\widetilde{P}_{k / k}^{\zeta}+Q_{k}^{\zeta}, \\
\widehat{\zeta}_{k+1 / k+1}=\widehat{\zeta}_{k+1 / k}+K_{k+1}^{\zeta}\left[\widetilde{r}_{k+1}-H_{k+1 / k} \widehat{\zeta}_{k / k}\right], \\
K_{k+1}^{\zeta}=P_{k+1 / k}^{\zeta} H_{k+1 / k}^{T}\left[H_{k+1 / k} P_{k+1 / k}^{\zeta} H_{k+1 / k}^{T}+\widetilde{S}_{k+1}\right]^{-1}, \\
P_{k+1 / k+1}^{\zeta}=\left[I-K_{k+1}^{\zeta} H_{k+1 / k}\right] P_{k+1 / k}^{\zeta} .
\end{gathered}
$$

Coupling equations are

$$
\begin{gathered}
W_{k}=A_{k} V_{k / k}+E_{k}, \\
V_{k+1 / k}=W_{k} P_{k / k}^{\zeta}\left[P_{k+1 / k}^{\zeta}\right]^{-1}, \\
H_{k+1 / k}=C_{k+1} V_{k+1 / k}, \\
V_{k+1 / k+1}=V_{k+1 / k}-\widetilde{K}_{k+1}^{x} H_{k+1 / k} .
\end{gathered}
$$

Compensated state and error covariance estimates are

$$
\begin{gathered}
\widehat{x}_{k+1 / k+1}=\widetilde{x}_{k+1 / k+1}+V_{k+1 / k+1} \widehat{\zeta}_{k+1 / k+1}, \\
P_{k+1 / k+1}=\widetilde{P}_{k+1 / k+1}^{x}+V_{k+1 / k+1} P_{k+1 / k+1}^{\zeta} V_{k+1 / k+1}^{T} .
\end{gathered}
$$

\section{Simulation Results}

Model. The adaptive MTSKF is applied to the longitudinal model of nonlinear B747 aircraft linearized around a trim point. The overall structure of the aircraft subject to actuator failures is given in Figure 2.

The model used in this study is a Boeing 747 aircraft, which is an intercontinental wide-body transport with four fan jet engines designed to operate from international airports. The nonlinear model of the aircraft given in [18], including the set of aerodynamic coefficients, is linearized around a trim point and then used in the simulations to assess the actuator fault isolation and identification performance of the adaptive MTSKF. The state space model of the linearized longitudinal dynamics of the $\mathrm{B} 747$ is as follows:

$$
\begin{gathered}
x_{k+1}=A x_{k}+B u_{k}+w_{k}^{x} \\
y_{k+1}=C x_{k+1}+v_{k+1},
\end{gathered}
$$

where

$$
\begin{gathered}
x_{k}=\left[\begin{array}{lllll}
\alpha & q & V & \theta & h
\end{array}\right]_{k}^{T}, \\
u_{k}=\left[\begin{array}{lll}
\delta_{e} & \delta_{s} & T_{c}
\end{array}\right]_{k}^{T},
\end{gathered}
$$

$\alpha$ is angle of attack (rad), $q$ is pitch rate $(\mathrm{rad} / \mathrm{sec}), V$ is true air speed $(\mathrm{m} / \mathrm{sec}), \theta$ is pitch angle $(\mathrm{rad}), h$ is altitude $(\mathrm{m}), \delta_{e}$ is elevator deflection (deg), $\delta_{s}$ is stabilizer deflection (rad), and $T_{c}$ is engine thrust $(\mathrm{N})$. Note that the states and the inputs are perturbations around the trim values.

The considered flight envelope is $\{(\alpha, V) \mid-2 \leq \alpha \leq$ 10 (deg), $150 \leq V \leq 250(\mathrm{~m} / \mathrm{sec})\}$ at an altitude of $7000 \mathrm{~m}$. Aerodynamic coefficients and their derivatives are calculated from the look-up tables (Esteban and Balas [1] and Shin et al. [19]). The trim condition is selected as $\{\alpha=\theta=0.93$ (deg), $V=230(\mathrm{~m} / \mathrm{sec}), \delta_{e}=0(\mathrm{deg}), \delta_{s}=0.0128(\mathrm{rad})$, and $T_{c}=41631$ (Newton) $\}$.

Throughout the simulations, the symbols seen in the figures are as follows: the state vector is $\left[\begin{array}{lllll}x^{1} & x^{2} & x^{3} & x^{4} & x^{5}\end{array}\right]^{T}=\left[\begin{array}{lllll}\alpha & q & V & \theta & h\end{array}\right]^{T}$ and $x_{\text {est }}^{i}(i=$ $1,2, \ldots, 5)$ is their estimations. The surface fault parameter vector is $\left[\begin{array}{llllll}\zeta^{1} & \zeta^{2} & \zeta^{3} & \zeta^{4} & \zeta^{5} & \zeta^{6}\end{array}\right]^{T}=\left[\begin{array}{llllll}\gamma^{1} & \gamma^{2} & \gamma^{3} & \beta^{1} & \beta^{2} & \beta^{3}\end{array}\right]^{T}$.

The data corresponding to the states and control inputs were collected from a Simulink model of the closed-loop nonlinear B747 model provided by Shin et al. [19]. The data were used in the adaptive MTSKF algorithm coded in Matlab for a sampling time of 0.05 seconds. The same results were also obtained by online identification settings in Simulink. The actuator faults are assumed to occur symmetrically, for example, right and left stabilizers, or right and left elevators are considered to move together.

Several experiments were performed as follows.

Experiment 1 (stuck fault in stabilizer at 0.05 (rad) at 20 seconds). Under the fault condition, simulation results for state and stuck fault estimation are shown in Figures 3 and 4 , respectively.

In Figure 3, it can be seen that the states are estimated correctly before and after occurrence of the stuck fault in the stabilizer.

From Figure 4, it can be concluded that a stuck actuator fault with magnitude 0.05 (rad) has occurred in the stabilizer because $\widehat{\zeta}^{2}=\widehat{\gamma}^{2}=-1$ and $\widehat{\zeta}^{5}=\widehat{\beta}^{2}=0.05$. When $\widehat{\gamma}$ becomes -1 , we can obtain the estimated stuck magnitude in the corresponding control channel.

The estimation error $\zeta^{6}-\widehat{\zeta}^{6}$ seems large, but, in fact, it is relatively small since the actual value for engine thrust is $41631(\mathrm{~N})$. Therefore, the estimation error in the engine thrust under the considered stuck magnitude is acceptable. 


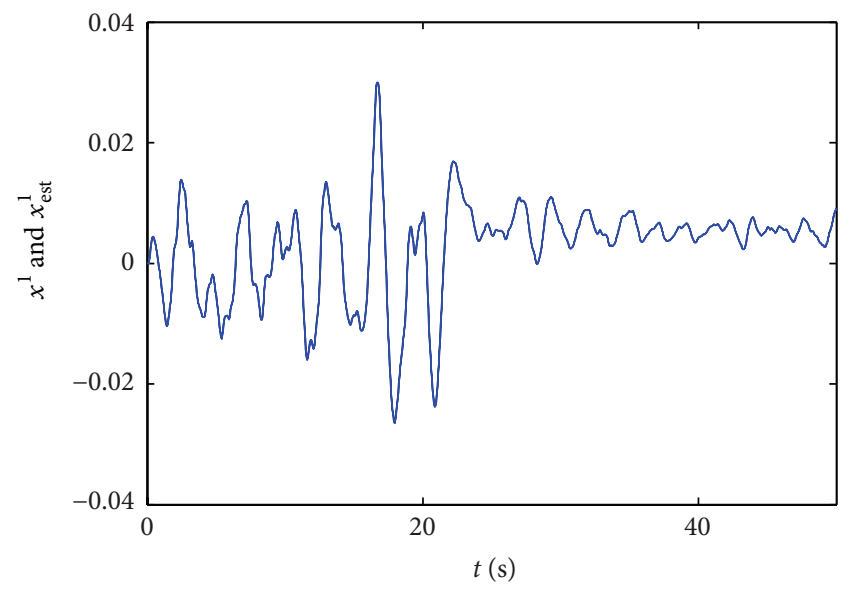

(a)

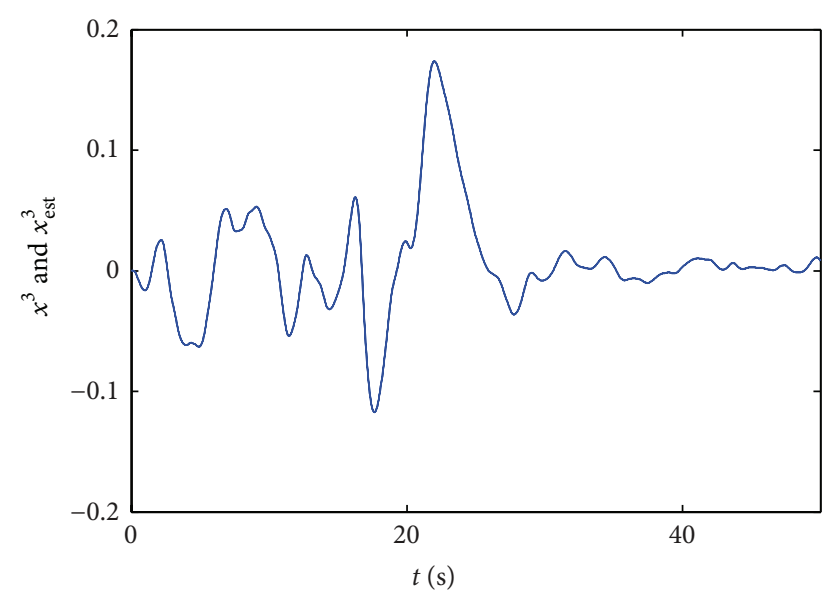

(c)

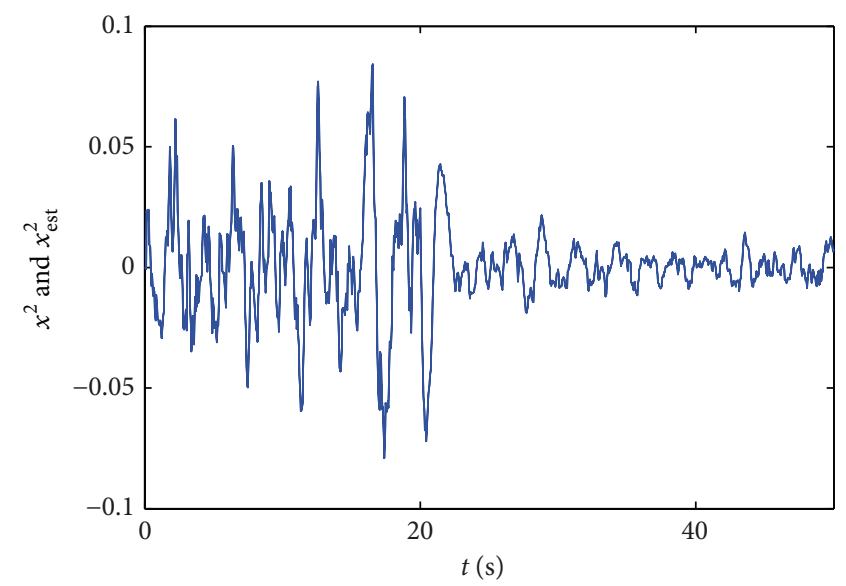

(b)

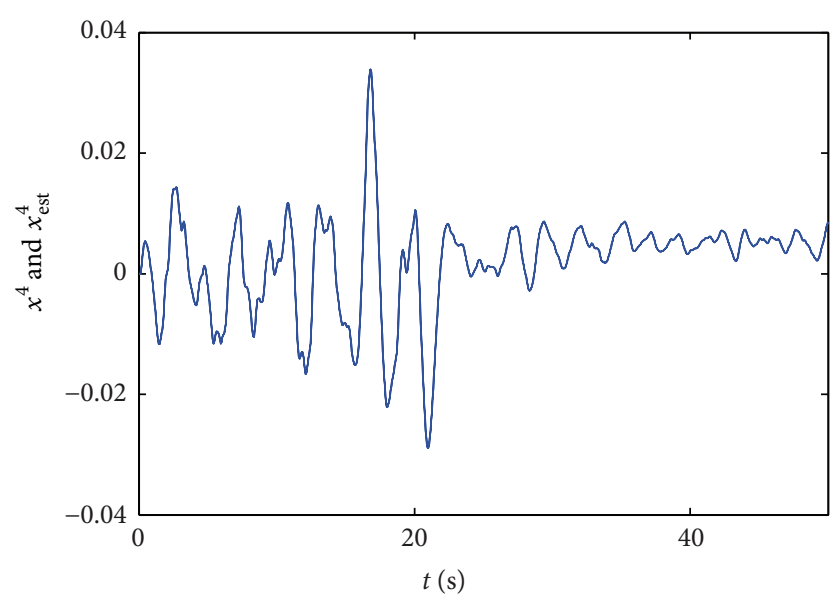

(d)

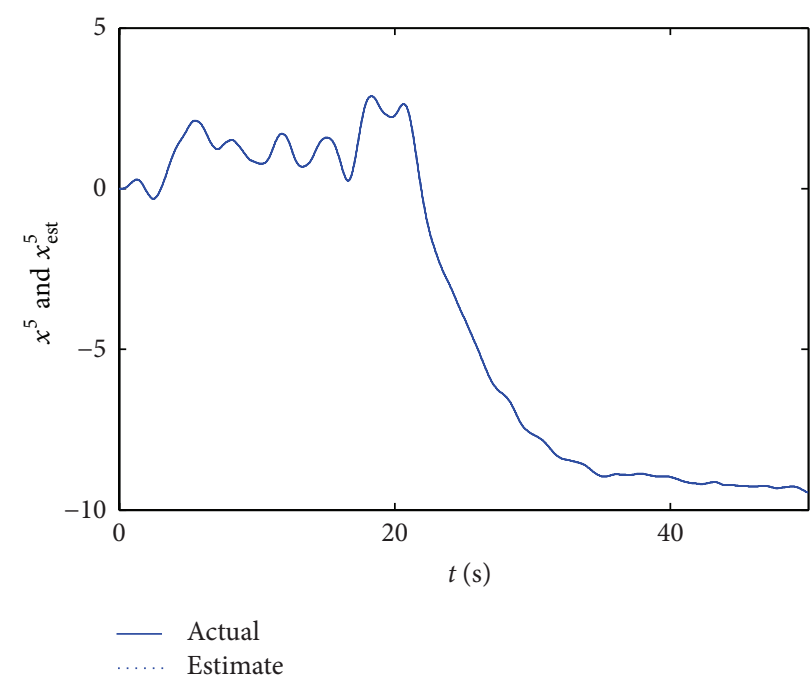

(e)

Figure 3: Actual state variables and estimations when a stuck fault in stabilizer occurred at 20 seconds. Since actual and estimated state variables are too close, two curves are seen as one curve. 


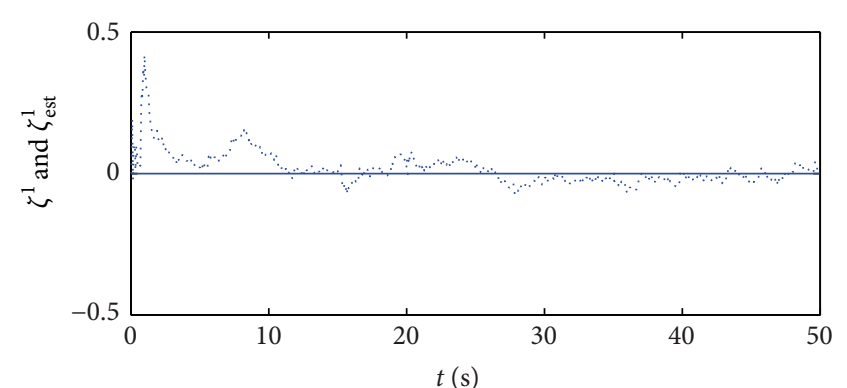

(a)

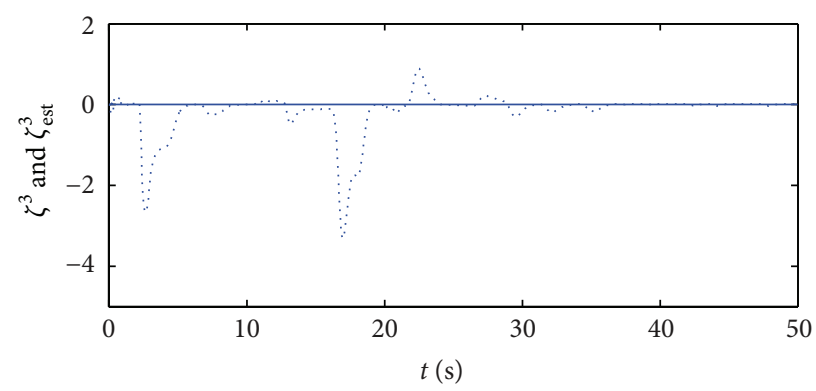

(c)

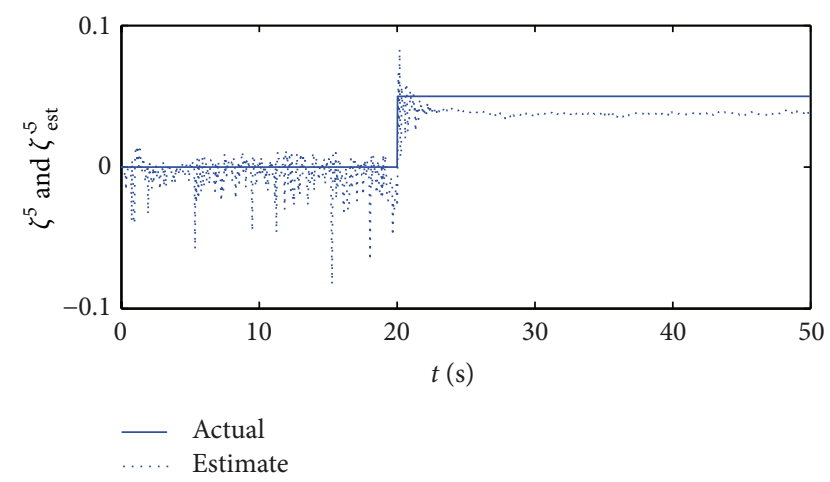

(e)

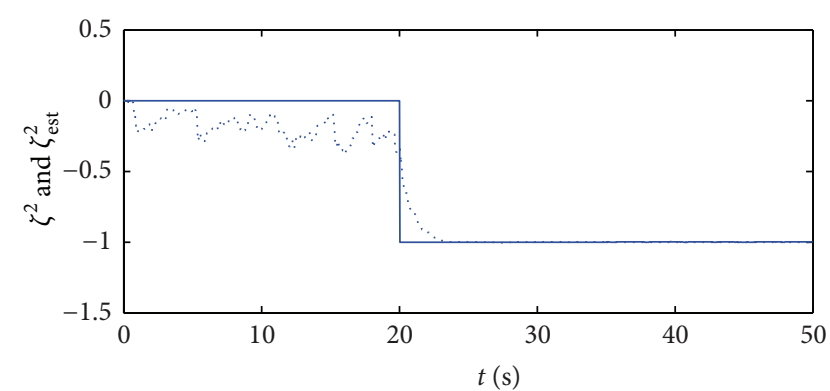

(b)

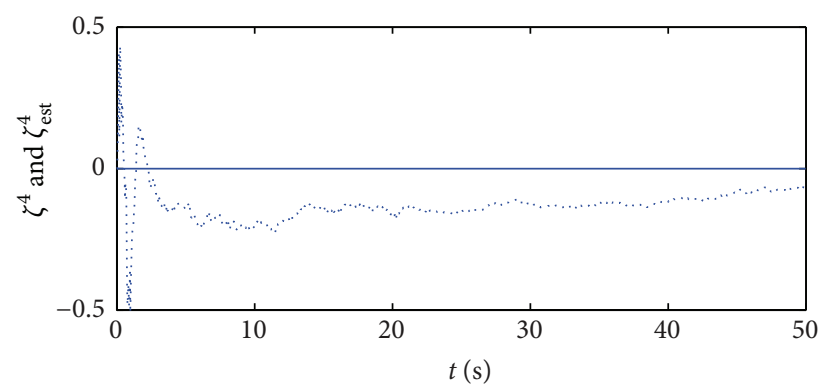

(d)

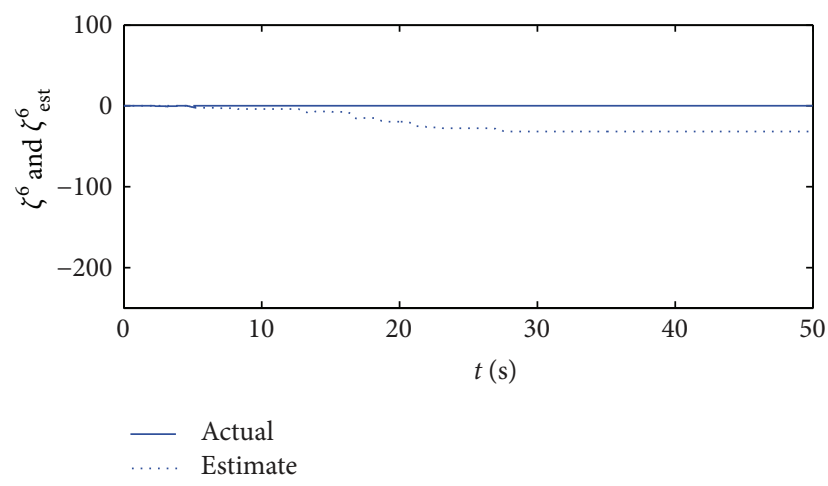

(f)

FIGURE 4: Actual inputs and estimations when a stuck actuator fault in stabilizer occurred at 20 seconds.

Experiment 2 (50\% partial loss in stabilizer at 20 seconds). Under the fault condition, simulation results for state and stuck fault estimation are shown in Figures 5 and 6, respectively.

In Figure 5, it can be seen that the states are estimated correctly before and after occurrence of the partial loss in the stabilizer. The operating point has not changed much.

In Figure 6, $\widehat{\zeta}^{2}=\widehat{\gamma}^{2}=-0.5$ and $\widehat{\zeta}^{5}=\widehat{\beta}^{2}=0$ are obtained, which means that $50 \%$ loss in stabilizer has occurred.

Experiment 3 (stuck fault in elevator at 0.0001 (rad) at 20 seconds). Due to the significant effect of elevator on the dynamics of the B747, the operating point has considerably changed due to the stuck fault in elevator. The adaptive MTSKF could not estimate the accurate stuck magnitude due to the deviation of the linearized model used in the adaptive MTSKF although the fault magnitude is small. However, satisfactory state estimates are obtained as shown in Figure 7. It is obvious that the trim point has changed after 20 seconds. The elevator stuck at trim has also been tested but the operating point has considerably changed again and the plant has become unstable. The filter is not successful in identifying stuck elevator faults. Therefore, the controller should be redesigned to take care of instability.

The operating point has changed severely. Since actual and estimated state variables are too close, two curves are seen as one curve. Although state estimation is highly accurate, the parameter estimation is not accurate. Therefore, the simulation results are not presented. To overcome this problem, a general two-stage extended Kalman filter (GTSEKF), which extends the linear general two-stage Kalman filter to nonlinear systems, may be proposed. As, in the linear one, the GTSEKF is derived mainly by applying the nonlinear twostage transformation to the well-known extended Kalman filter (EKF). A nonlinear filter for estimating constant parameters in dynamic systems is presented in [20]. 


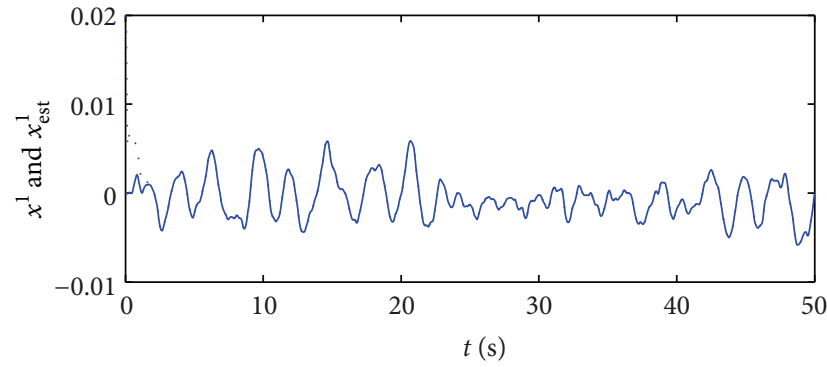

(a)

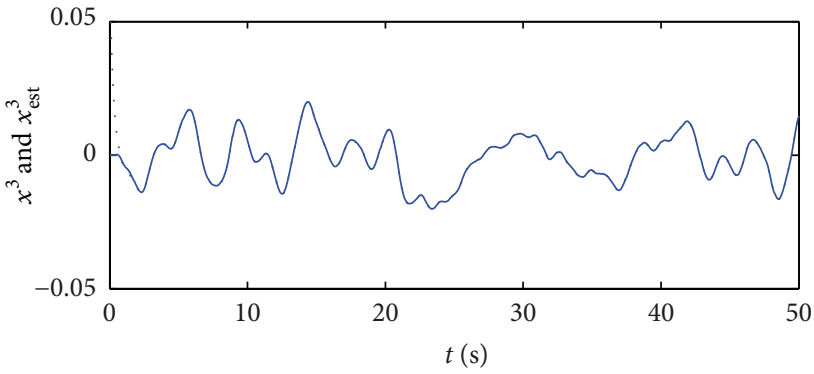

(c)

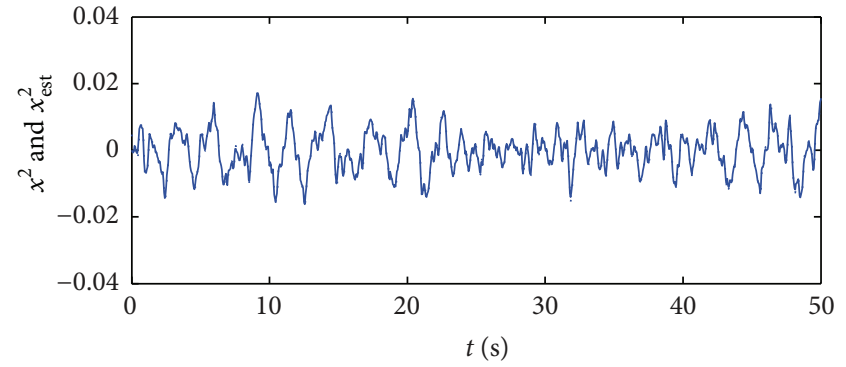

(b)

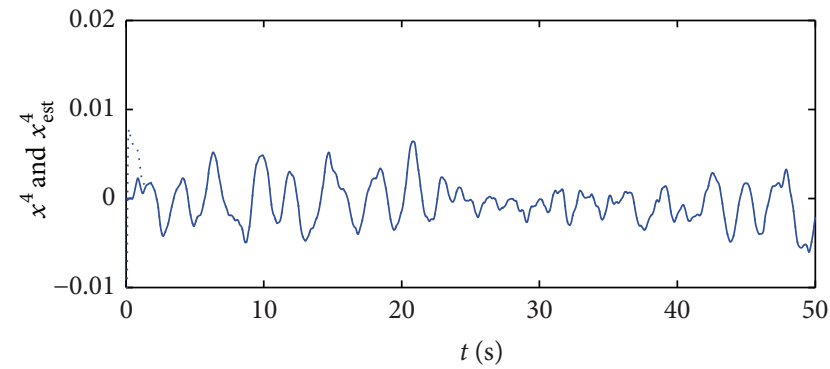

(d)

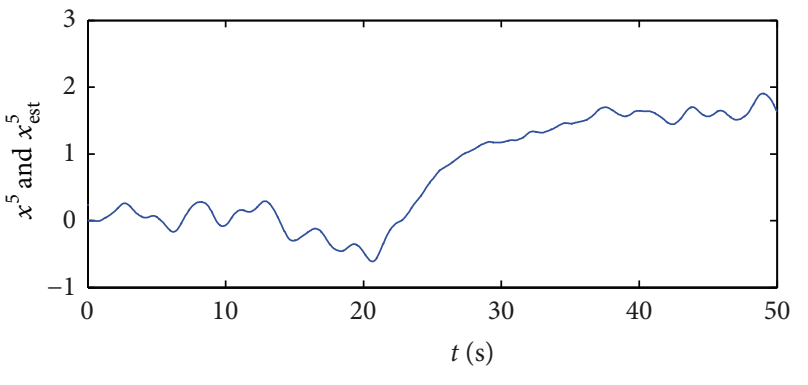

Actual

Estimate

(e)

Figure 5: Actual state variables and estimations when 50\% partial loss in stabilizer occurred at 20 seconds. Since actual and estimated state variables are too close, two curves are seen as one curve.

It has been observed from the simulations that actuator dynamics play very important role in the identification of the parameters. The excitation and control signals need to be prefiltered to be able to obtain accurate estimation results under the assumption that the adaptive MTSKF does not have actuator model. It has also been observed that larger $Q^{\zeta}$ makes the convergence faster.

\section{Conclusions}

In this paper, an adaptive MTSKF was designed and used to estimate the reduction of control effectiveness and the magnitude of stuck faults for a nonlinear B747 model. Some simulation results on fault parameter estimation performed on a nonlinear model of the longitudinal motion of the aircraft were presented. The linear adaptive MTSKF is successful in identifying the magnitude of the stuck fault and the percentage of the partial loss in the stabilizer. Control inputs have been excited by a pseudorandom noise such that successful estimation of stuck magnitudes and loss of effectiveness can be achieved for a limited linear region. The study showed that the linear adaptive MTSKF is successful in identifying low degree stuck fault and partial loss in the stabilizer. However, the plant nonlinearity must be included in the design model of the filter to allow the parameters represent more severe faults to be correctly estimated. While this study focuses on Boeing 747 aircraft, the estimation procedure may also have applications for other aircraft types and to the military and space sectors.

\section{Nomenclature}

FDI: Fault Detection and Identification

ARC: Adaptive Reconfigurable Control

LMI: Linear Matrix Inequality

TSKF: Two-stage Kalman filter

FDD: Fault Detection and Diagnosis 


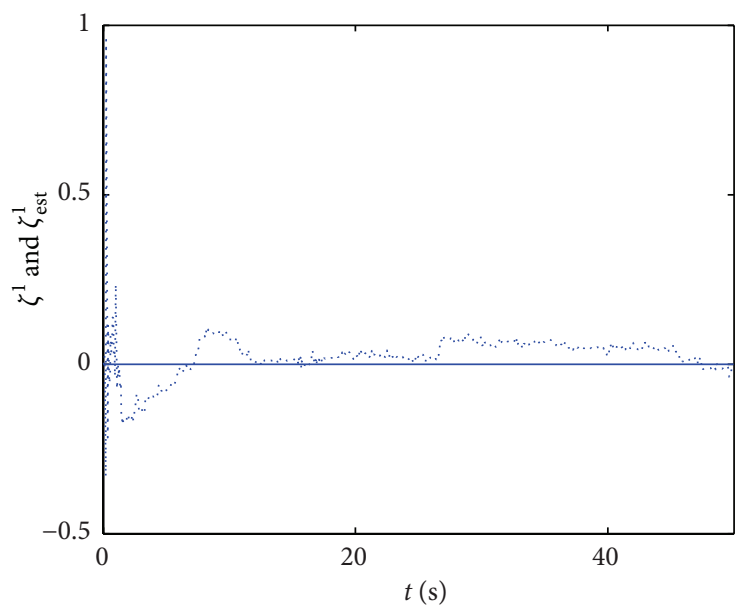

(a)

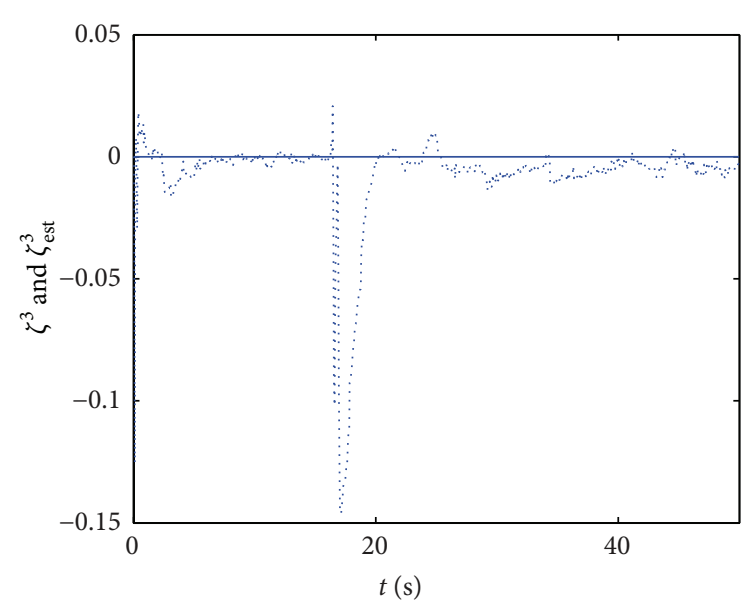

(c)

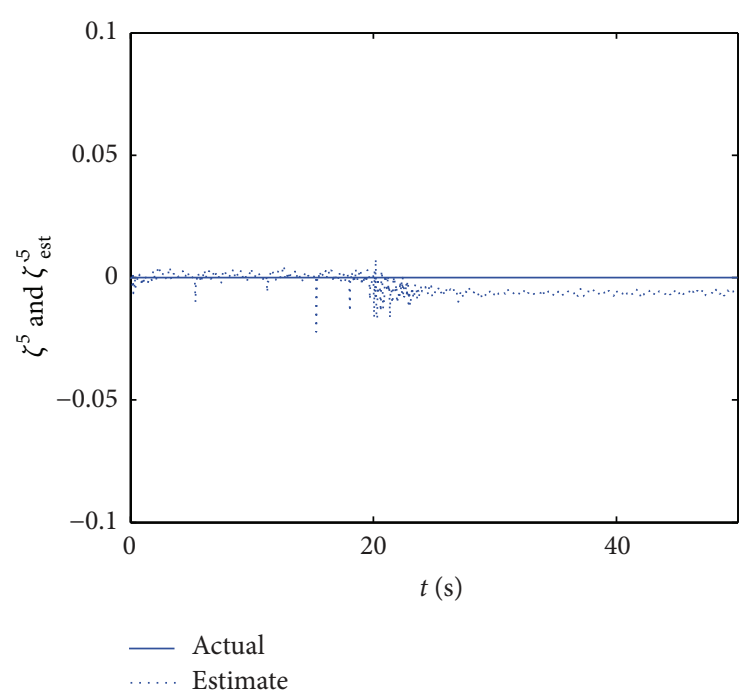

(e)

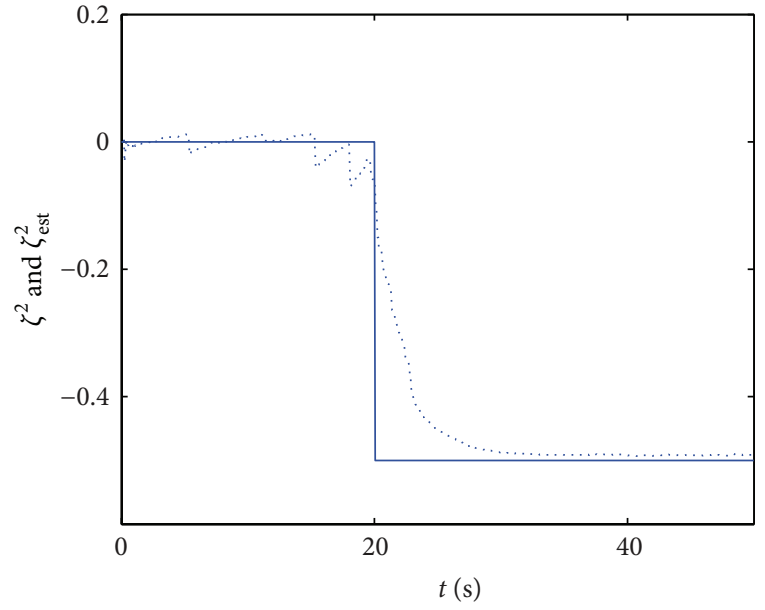

(b)

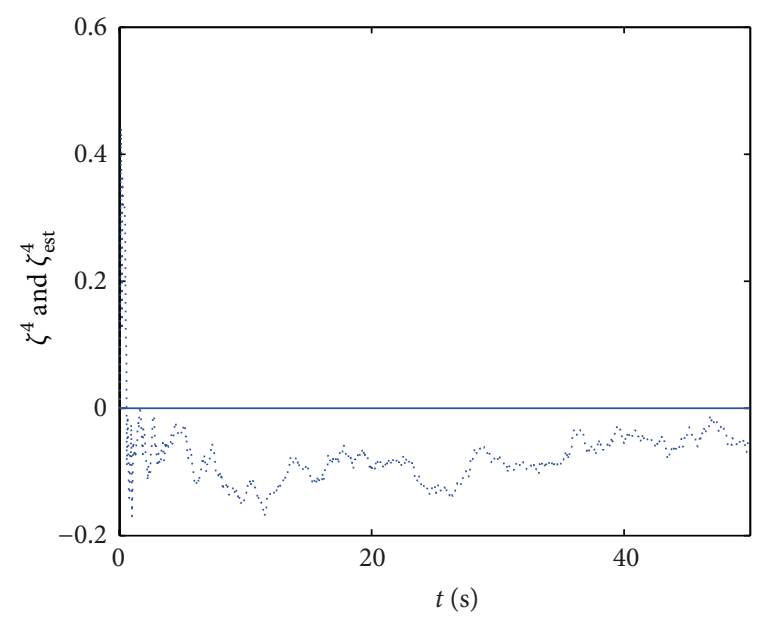

(d)

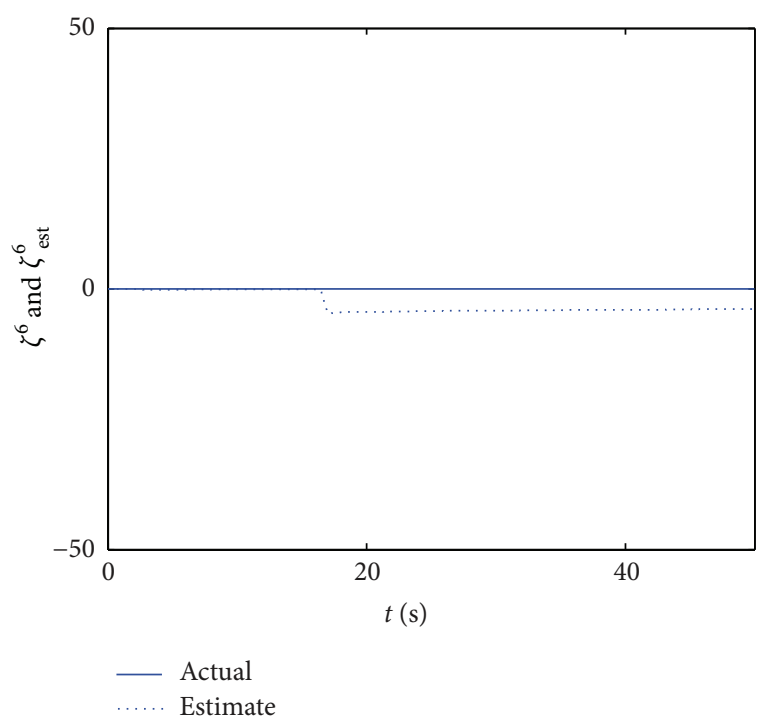

(f)

Figure 6: Actual inputs and estimations when $50 \%$ partial loss in stabilizer occurred at 20 seconds. 


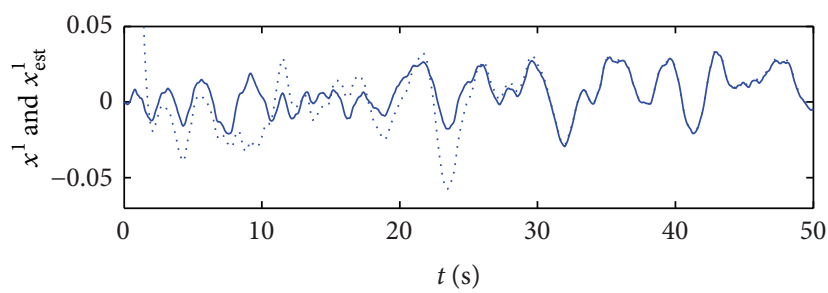

(a)

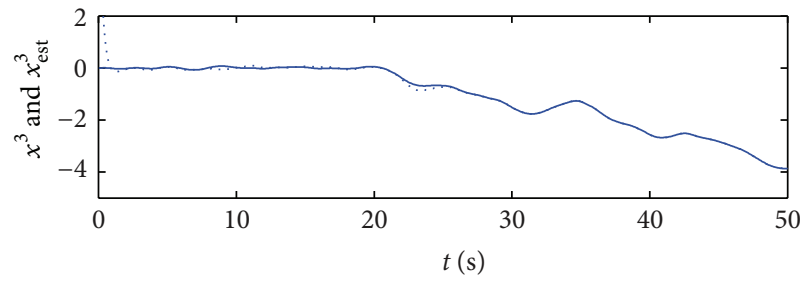

(c)

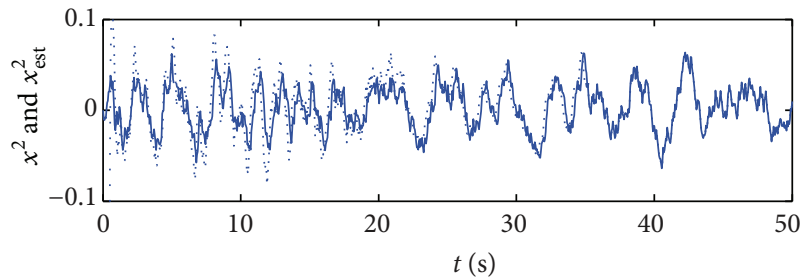

(b)

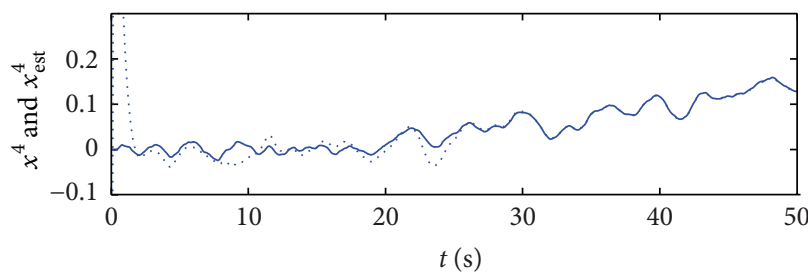

(d)

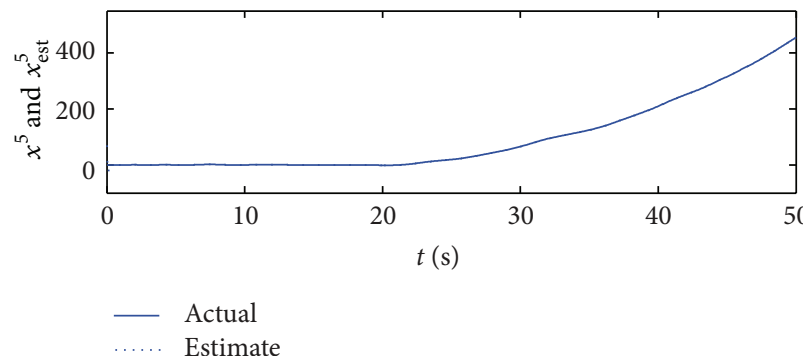

(e)

Figure 7: Actual state variables and estimations when a stuck fault in elevator occurred at 20 seconds.

MTSKF: $\quad$ Modified Two-Stage Kalman filter

$x_{k}, u_{k}$, and $y_{k+1}$ : The state, control input, and output variables

$\gamma_{k}$ and $\beta_{k}$ : Bias vectors representing faults entering

$w^{x}, w^{\zeta}: \quad$ Noise sequences

$\alpha: \quad$ Angle of attack

q: $\quad$ Pitch rate

$V: \quad$ True air speed

$\theta: \quad$ Pitch angle

$h$ : Altitude

$\delta_{e}: \quad$ Elevator deflection

$\delta_{s}: \quad$ Stabilizer deflection

$T_{c}$ : $\quad$ Engine thrust.

\section{Conflict of Interests}

The authors declare that there is no conflict of interests regarding the publication of this paper.

\section{Acknowledgment}

This study was partially supported by NASA Langley NNX08AC65A contract entitled "Fault Diagnosis, Prognosis and Reliable Flight Envelope Assessment."

\section{References}

[1] A. M. Esteban and G. Balas, "Linear parameter varying modelling of the Boeing 747-100/200 longitudinal motion," in Proceedings of the AIAA Guidance, Navigation and Control Conference, vol. AIAA-01-4347, American Institute of Aeronautics and Astronautics, Montreal, Canada, 2001.

[2] N. E. Wu, Y. Zhang, and K. Zhou, "Detection, estimation, and accommodation of loss of control effectiveness," International Journal of Adaptive Control and Signal Processing, vol. 14, no. 7, pp. 775-795, 2000.

[3] J.-Y. Shin, N. E. Wu, and Y. Zhang, "Dynamic flight envelope assessment and prediction," proposal to NASA Aviation Safety Program-Integrated Resilient Aircraft Control (IRAC), NASA, 2007.

[4] G. Vachtsevanos, L. Tang, G. Drozeski, and L. Gutierrez, "From mission planning to flight control of unmanned aerial vehicles: strategies and implementation tools," Annual Reviews in Control, vol. 29, no. 1, pp. 101-115, 2005.

[5] W. Chen and J. Jiang, "Fault tolerant control against stuck actuator faults," IEE Proceedings, vol. 152, no. 2, pp. 138-146, 2005.

[6] J. D. Boskovic and R. K. Mehra, "A robust adaptive reconfigurable flight control scheme for accommodation of control effector failures," in Proceedings of the American Control Conference, pp. 1127-1132, Arlington, Va, USA, June 2001. 
[7] B. Jiang and F. N. Chowdhury, "Fault estimation and accommodation for linear MIMO discrete-time systems," IEEE Transactions on Control Systems Technology, vol. 13, no. 3, pp. 493-499, 2005.

[8] G. Yang and H. Wang, "Fault detection for a class of state feedback control systems," in Proceedings of the American Control Conference, pp. 5784-5789, New York, NY, USA, July 2007.

[9] D. Wang and K. Lum, "Adaptive unknown input observer approach for aircraft actuator fault detection and isolation," International Journal of Adaptive Control and Signal Processing, vol. 21, no. 1, pp. 31-48, 2007.

[10] D. Wang, "Private communication," 2008.

[11] J. Y. Keller and M. Darouach, "Optimal two-stage Kalman filter in the presence of random bias," Automatica, vol. 33, no. 9, pp. 1745-1748, 1997.

[12] J. E. Parkum, N. K. Poulsen, and J. Holst, "Recursive forgetting algorithms," International Journal of Control, vol. 55, no. 1, pp. 109-128, 1992.

[13] Y. M. Zhang and J. Jiang, "Active fault-tolerant control system against partial actuator failures," IEE Proceedings, vol. 149, no. 1, pp. 95-104, 2002.

[14] J.-Y. Shin, N. E. Wu, and C. Belcastro, "Adaptive linear parameter varying control synthesis for actuator failure," Journal of Guidance, Control, and Dynamics, vol. 27, no. 5, pp. 787-794, 2004.

[15] M. H. Amoozgar, A. Chamseddine, and Y. Zhang, "Experimental test of a two-stage Kalman filter for actuator fault detection and diagnosis of an unmanned quadrotor helicopter," Journal of Intelligent Robot Systems, vol. 70, no. 1, pp. 107-117, 2013.

[16] Ch. Hajiyev, "Two-stage Kalman filter-based actuator/surface fault identification and reconfigurable control applied to F-16 fighter dynamics," International Journal of Adaptive Control and Signal Processing, vol. 27, no. 9, pp. 755-770, 2013.

[17] B. Jiang, Y. Guo, and P. Shi, "Adaptive reconfiguration scheme for flight control systems," Proceedings of the Institution of Mechanical Engineers I, vol. 224, no. 6, pp. 713-723, 2010.

[18] A. Marcos, S. Ganguli, and G. J. Balas, "Application of $\mathrm{H}_{\mathrm{inf}}$ fault detection and isolation to a Boeing 747-100/200 aircraft," in Proceedings of the AIAA Guidance, Navigation, and Control Conference, vol. AIAA-2002-4944, Monterey, Calif, USA, August 2002.

[19] J.-Y. Shin, C. Belcastro, and T. Khong, "Closed-loop evaluation of an integrated failure identification and fault tolerant control system for a transport aircraft," in Proceedings of the AIAA Guidance, Navigation and Control Conference and Exhibit, vol. AIAA-2006-6310, pp. 1973-1994, Keystone, Colo, USA, August 2006.

[20] C. Hsieh, "General two-stage extended Kalman filters," IEEE Transactions on Automatic Control, vol. 48, no. 2, pp. 289-293, 2003. 

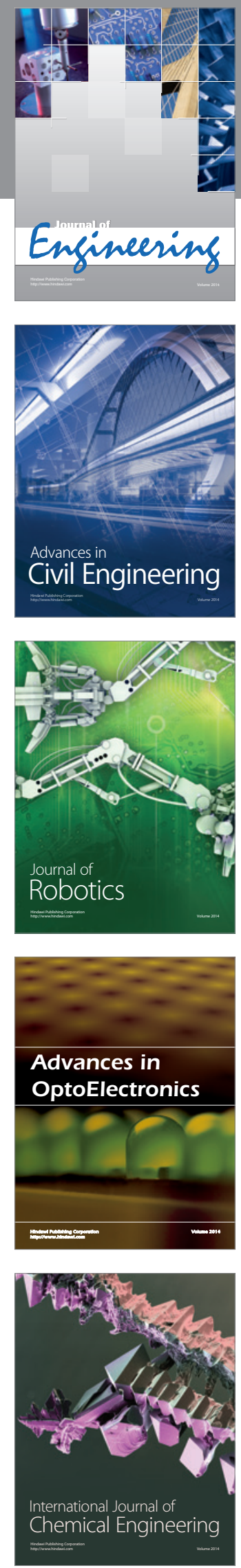

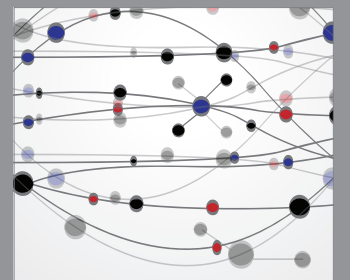

The Scientific World Journal
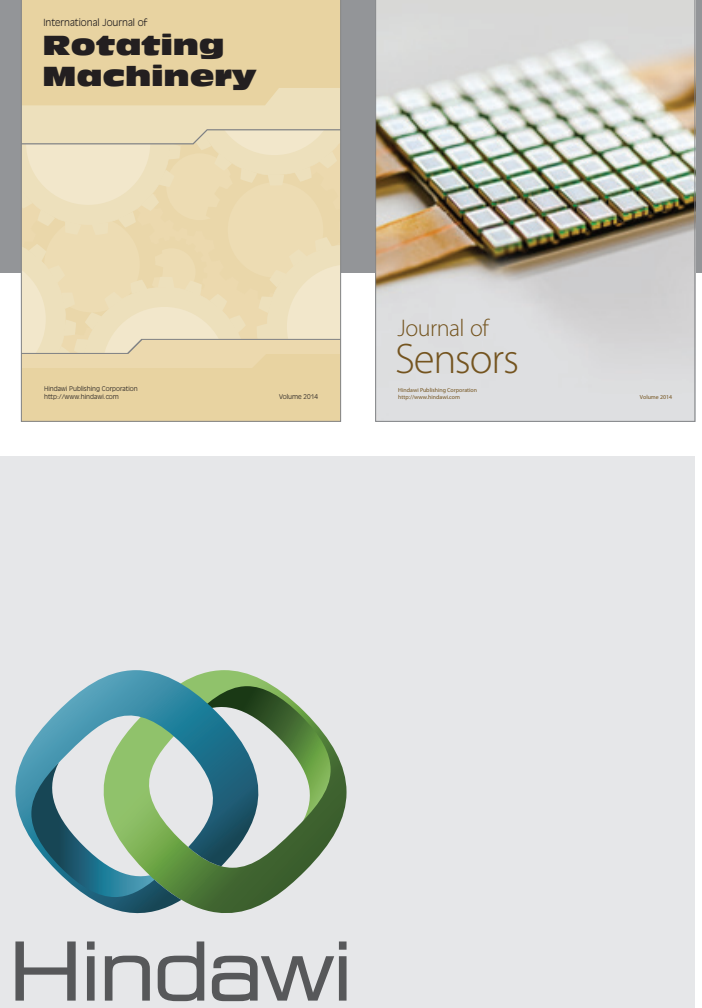

Submit your manuscripts at http://www.hindawi.com
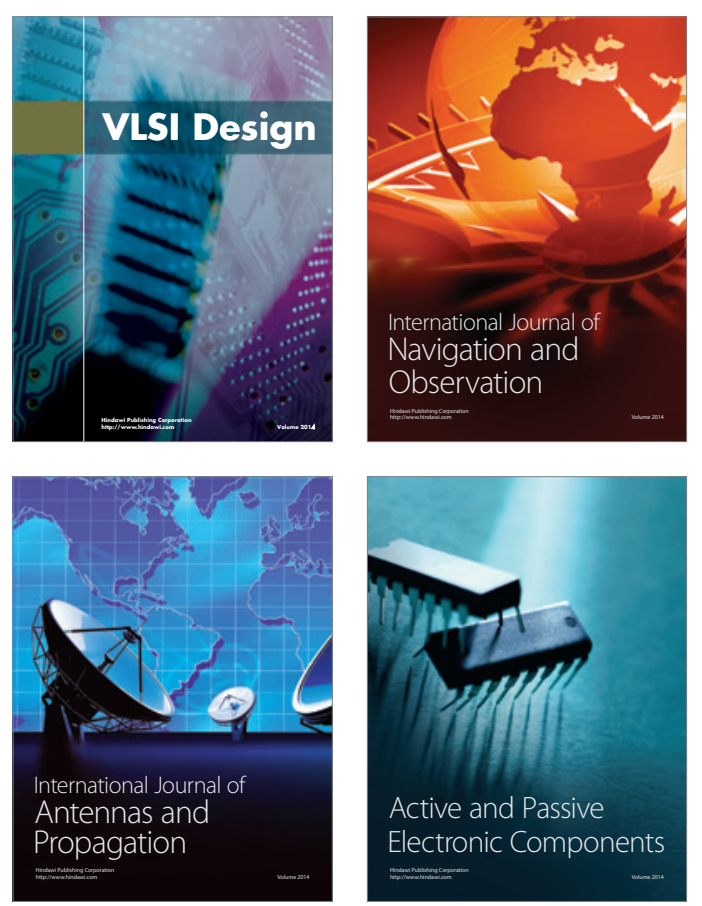
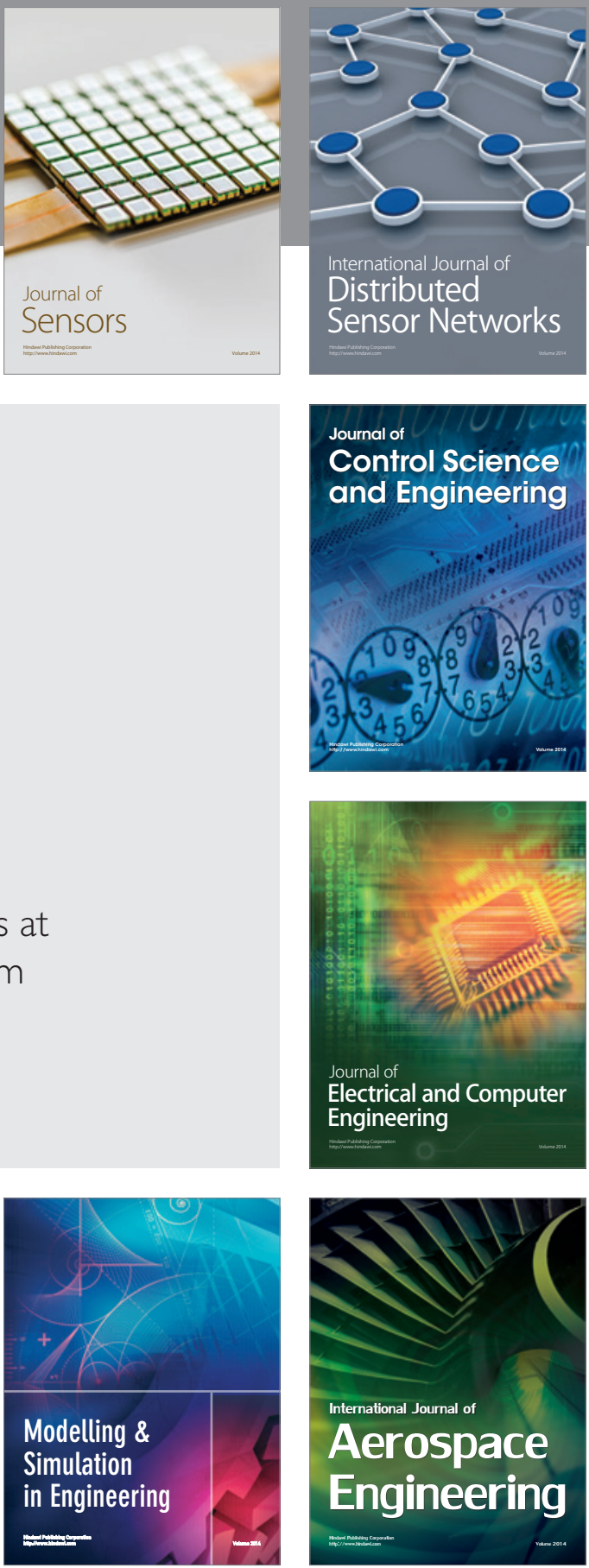

Journal of

Control Science

and Engineering
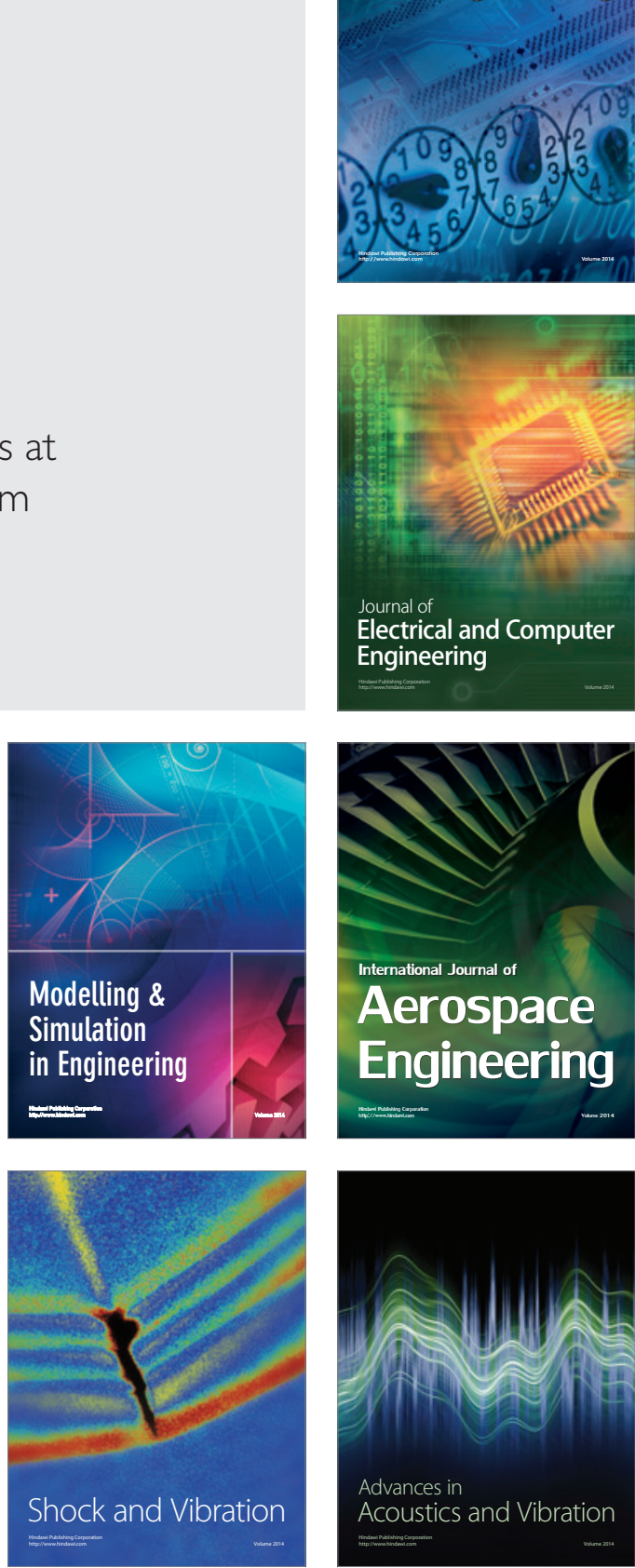